\title{
Nonlinear Optical Properties of Polyynes: An Experimental Prediction for Carbyne
}

\author{
Nisha R. Agarwal, ${ }^{*},{ }^{\dagger} \|$ Andrea Lucotti, ${ }^{\dagger}$ Matteo Tommasini, ${ }^{\dagger}$ Wesley A. Chalifoux, ${ }^{\ddagger}$ and Rik R. Tykwinski ${ }^{\S}$ \\ ${ }^{\dagger}$ Dipartimento di Chimica, Materiali e Ingegneria Chimica “Giulio Natta”, Politecnico di Milano, Piazza Leonardo da Vinci 32,20133 \\ Milano, Italy \\ ${ }^{\ddagger}$ Department of Chemistry, University of Nevada, Reno, Nevada 89557, United States \\ ${ }^{\S}$ Department of Chemistry and Pharmacy \& Interdisciplinary Center of Molecular Materials (ICMM), University of \\ Erlangen-Nuremberg, Henkestrasse 42, 91054 Erlangen, Germany
}

Supporting Information

ABSTRACT: We present the experimental determination of the vibrational contribution to molecular second hyperpolarizability $\left(\gamma_{\mathrm{vib}}\right)$ of very long polyynes that have been recently made available thanks to progress in chemical synthesis. Based on a simple theoretical model, the available experimental data allow estimating the asymptotic behavior of the vibrational contribution to molecular hyperpolarizability for increasing chain length.

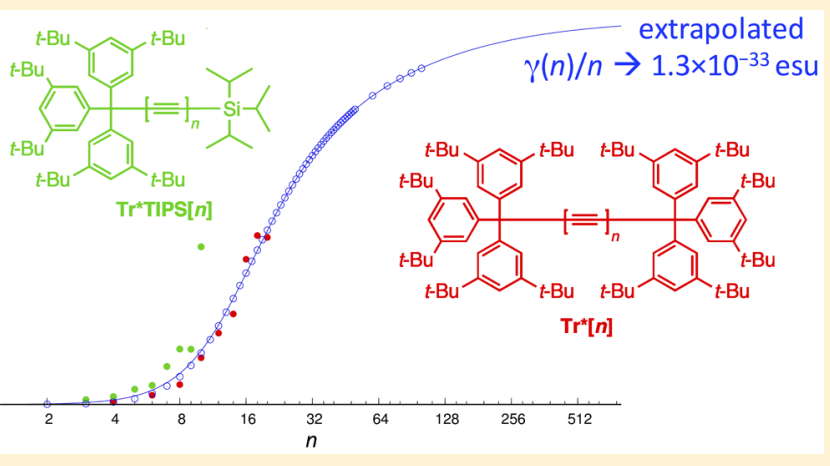

\section{INTRODUCTION}

Since the early 1990s, when fullerenes became accessible in macroscopic quantities thanks to Krätchmer and Huffman's synthetic protocol, ${ }^{1}$ the carbon allotropes have become increasingly more prominent contributors in materials science. $^{2-6}$ For example, diamond nanowires can now be formed from the bottom up ${ }^{7}$ or the top down approach, ${ }^{8}$ fullerenes continue to function as the primary acceptor in solar cells, ${ }^{9}$ carbon nanotubes inch closer to realistic use semiconductor devices, ${ }^{10}$ and graphene is arguably the prominent candidate to replace indium tin oxide (ITO) in transparent electrodes. $^{11}$ New and yet undiscovered synthetic carbon allotropes are also targets of study, such as graphyne, which could show properties that rival or even surpass those of graphene. ${ }^{12}$ Also in this category of "emerging" allotropes is carbyne, a material composed of sp-hybridized carbon atoms. ${ }^{6,13}$ While there have been claims of carbyne formation over the years, ${ }^{14-17}$ definitive characterization of carbyne is yet to be achieved, at least in the opinion of the authors. While carbyne may not yet exist, chemists, physicists, and materials scientists have worked hard to explore its potential properties, often through the construction of model compounds, i.e., oligomers with defined structure and length. ${ }^{18}$ With a series of structurally related compounds in hand, changes observed for properties as a function of length can then be extrapolated to infinite length, offering a glimpse of what constitutes carbyne. ${ }^{19}$

The formation of sp-hybridized carbon chains has been achieved in solution through numerous chemical and physical methods, ${ }^{14-17,20-23}$ and they have been embedded in the interior of carbon nanotubes ${ }^{24,25}$ and trapped in an inert gas matrix at low temperature. ${ }^{26,27}$ The effectiveness of such methods is, however, severely limited by the thermodynamic instability of sp-hybridized carbon chains in the condensed state. $^{28,29}$ This reactivity can readily lead to cross-linking and the formation of $\mathrm{sp}^{2}$ - and $\mathrm{sp}^{3}$-carbon linkages, which hinders more extensive investigations of physical, optoelectronic, and spectroscopic properties. $^{28,30}$

In order to stabilize polyynes and facilitate the study of their properties, we $\mathrm{e}^{31-37}$ and others ${ }^{38-44}$ have focused on the incorporation of sterically bulky groups at the two termini of the polyyne chain. This strategy has achieved varying levels of success over the years, and recently culminated in the formation of the "super trityl" series of polyynes ( $\operatorname{Tr}^{*}[\boldsymbol{n}]$, Figure 1), which incorporate up to 44 contiguous sp-hybridized carbon atoms $\left(\operatorname{Tr}^{*}[22]\right){ }^{19}$ This same study also provided unsymmetrical polyynes with up to 11 consecutive carbon-carbon triple bonds, namely Tr*TIPS[11] (Figure 1).

The realization of the $\operatorname{Tr}^{*}[n]$ and $\operatorname{Tr}{ }^{*} \operatorname{TIPS}[n]$ series of molecules offered opportunities to explore the cubic nonlinear optical (NLO) properties of very long symmetrical and unsymmetrical polyynes, as well as the unprecedented chance to predict the NLO characteristics of carbyne. Several studies have suggested that polyynes and carbyne have great potential as NLO materials, due to their linear structure and $\pi$-electron

Received: March 24, 2016

Revised: April 28, 2016

Published: May 2, 2016 


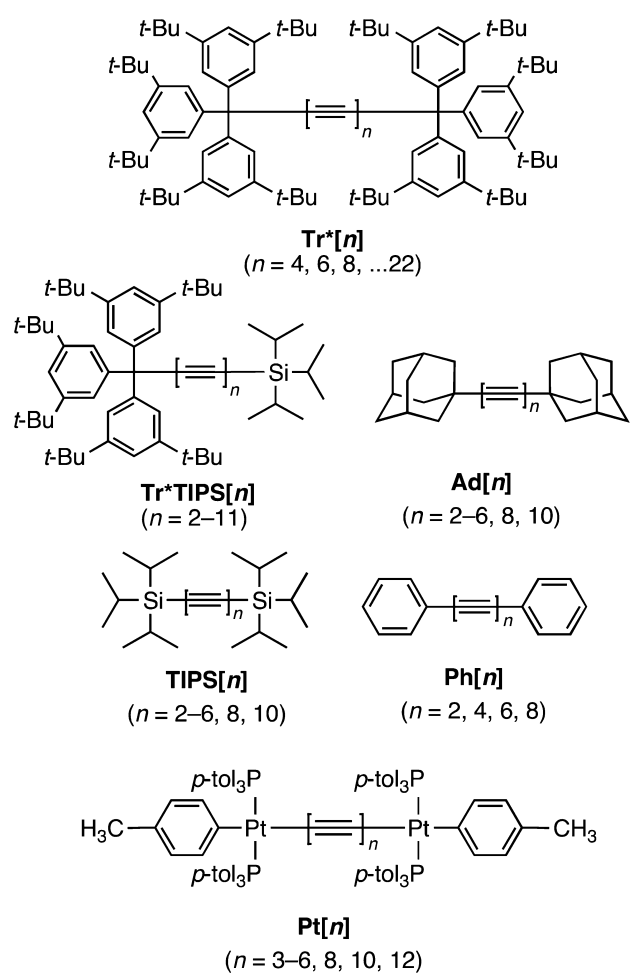

Figure 1. Molecular structures of polyynes discussed (or cited) in this work: supertrityl $\left(\operatorname{Tr}^{*}[n]\right)$, supertrityl-triisopropylsilyl ( $\operatorname{Tr}^{*}$ TIPS$[n])$, adamantyl $(\operatorname{Ad}[n])$, triisopropylsilyl (TIPS $[n])$, phenyl $(\mathbf{P h}[n])$, and platinum $(\mathbf{P t}[n]$ ) end-capped (where $n$ is the number of repeat units, i.e., $-\mathrm{C} \equiv \mathrm{C}-$ units).

delocalization, which is independent of bond rotation. ${ }^{37,45-52}$ For example, it has been recently reported that the vibrational component to the second hyperpolarizability $\gamma_{\text {vib }}$ values for the $\operatorname{Ad}[\boldsymbol{n}]$ polyynes $^{52}$ (measured by quantitative Raman spectroscopic analysis) are of the same order of magnitude as $\gamma_{\text {ele }}$ values found for TIPS $[\boldsymbol{n}]$ polyynes ${ }^{37,49}$ (measured by the differential optical Kerr effect (DOKE) technique ${ }^{53}$ ). These works have also suggested that the $\gamma_{\text {ele }}$-values for TIPS $[n]$ polyynes approach the theoretical limit discussed by Kuzyk based on a power law analysis. ${ }^{54}$

In the work reported herein, we outline trends in the molecular second hyperpolarizability for the symmetrical supertrityl $\left(\operatorname{Tr}^{*}[n]\right)$ polyynes and the unsymmetrical supertrityl-triisopropylsilyl (Tr*TIPS $[n]$ ) polyynes, as measured by quantitative Raman spectroscopic analysis. The influence of a polarized structure in the unsymmetrical Tr*TIPS $[n]$ polyynes is described by a comparison of the two series. Furthermore, we attempt to describe a global perspective of molecular second hyperpolarizability as a function of molecular length, using the NLO data available. Finally, we give an experiment-based prediction for the allotrope carbyne.

\section{EXPERIMENTAL SECTION}

$\operatorname{Tr}{ }^{*}[n]$ and $\operatorname{Tr}{ }^{*} \operatorname{TIPS}[n]$ polyynes were synthesized as reported in ref 19. Raman spectra were recorded using a Nicolet NXR 9650 FT-Raman equipped with a $\mathrm{Nd}-\mathrm{VO}_{4}$ laser providing a $1064 \mathrm{~nm}$ excitation line, an InGaAs detector, and a liquid nitrogen cooled germanium detector. In order to provide a good signal-to-noise ratio in the Raman spectra, solution measurements were done in reasonably concentrated solutions of the polyyne sample dissolved in toluene, in the range of
0.17-7.6 mM. Raman spectra were recorded using unpolarized light in the backscattering geometry, and the resolution is 4 $\mathrm{cm}^{-1}$ for all spectra described in this report. The infrared excitation wavelength was $1064 \mathrm{~nm}$, this ensured that there was not resonance enhancement of the vibrational contributions to the second hyperpolarizabilities; i.e., all values reported can be considered nonresonant since the electronic absorptions of polyynes $\left(\lambda_{\max }\right)$ are found in the violet-ultraviolet region of the spectrum, far from the $1064 \mathrm{~nm}$ excitation line (see Table 1).

Table 1. Summary of Second Hyperpolarizabilities $\left(10^{-36}\right.$ esu) and $\lambda_{\max }$ Values (nm, in Parentheses) for Polyynes $\operatorname{Tr}^{*}[n], \operatorname{Tr}^{*} \operatorname{TIPS}[n], \operatorname{Ad}[n]$, and TIPS $[n]$

\begin{tabular}{|c|c|c|c|c|}
\hline$n$ & $\gamma_{\operatorname{Tr}^{*}[n]}(\mathrm{esu})^{a}$ & $\begin{array}{c}\gamma_{\operatorname{Tr} * \operatorname{TIPS}[n]} \\
(\mathrm{esu})^{a}\end{array}$ & $\gamma_{\mathrm{Ad}[n]}(\mathrm{esu})^{c}$ & $\gamma_{\operatorname{TIPS}[n]}(\mathrm{esu})^{b}$ \\
\hline 2 & - & - & $2.87-$ & $2.75-$ \\
\hline 3 & - & $\begin{array}{c}48.5 \\
(<250 \mathrm{~nm})\end{array}$ & $\begin{array}{c}14.6 \\
(<250 \mathrm{~nm})\end{array}$ & $7.02(234 \mathrm{~nm})$ \\
\hline 4 & $47.4(268 \mathrm{~nm})$ & $106(264 \mathrm{~nm})$ & $43.0(247 \mathrm{~nm})$ & $12.5(260 \mathrm{~nm})$ \\
\hline 5 & - & $261(288 \mathrm{~nm})$ & $83.4(272 \mathrm{~nm})$ & $34.5(284 \mathrm{~nm})$ \\
\hline 6 & $190(310 \mathrm{~nm})$ & $393(310 \mathrm{~nm})$ & $116(295 \mathrm{~nm})$ & $64.5(304 \mathrm{~nm})$ \\
\hline 7 & - & $914(324 \mathrm{~nm})$ & - & - \\
\hline 8 & $547(347 \mathrm{~nm})$ & $1520(343 \mathrm{~nm})$ & $505(335 \mathrm{~nm})$ & $238(339 \mathrm{~nm})$ \\
\hline 9 & - & $1710(359 \mathrm{~nm})$ & - & - \\
\hline 10 & $1600(376 \mathrm{~nm})$ & $5420(373 \mathrm{~nm})$ & $935(367 \mathrm{~nm})$ & $646(369 \mathrm{~nm})$ \\
\hline 12 & $2940(400 \mathrm{~nm})$ & - & - & - \\
\hline 14 & $4360(419 \mathrm{~nm})$ & - & - & - \\
\hline 16 & $7980(432 \mathrm{~nm})$ & - & - & - \\
\hline 18 & $\begin{array}{l}10500 \\
(443 \mathrm{~nm})\end{array}$ & - & - & - \\
\hline 20 & $\begin{array}{l}11500 \\
(451 \mathrm{~nm})\end{array}$ & - & - & - \\
\hline
\end{tabular}

${ }^{a}$ As measured in the present study by absolute Raman intensity measurements in toluene; UV-vis spectra measured in hexanes. ${ }^{b} \mathrm{As}$ measured by absolute Raman intensity measurements in THF; UVvis spectra measured in hexanes, ref $52 .{ }^{c}$ As measured by the differential optical Kerr effect (DOKE) at $800 \mathrm{~nm}$ in THF; UV-vis spectra measured in hexanes, ref 37.

For the spectra reported in Figure 2, power at the sample was on the order of $0.6 \mathrm{~W}$ for $n<5$ and $0.3 \mathrm{~W}$ for longer polyynes on a laser spot diameter of approximately $150 \mu \mathrm{m}$; 2048 scans were accumulated for each spectrum, for a total measurement time of about $1 \mathrm{~h}$. The approach adopted here for the experimental determination of NLO properties proceeds through the measurement of Raman intensities, following the procedure illustrated in ref 52. Full experimental details, which also apply to the present work, are reported in the Supporting Information of ref 52 .

\section{RESULTS AND DISCUSSION}

Nonlinear Optical Determination. The second hyperpolarizability $\gamma$ is related to the cubic contribution to the molecular dipole change with respect to the applied electric field:

$$
\mu_{a}=\mu_{a}^{0}+\sum_{b} \alpha_{a b} E^{b}+\frac{1}{2 !} \sum_{b c} \beta_{a b c} E^{b} E^{c}+\frac{1}{3 !} \sum_{b c d} \gamma_{a b c d} E^{b} E^{c} E^{d}
$$

In addition to the importance of electronic contributions to $\gamma$, it has been determined for many $\pi$-conjugated materials that contributions due to changes of molecular geometry upon applied fields also play a leading role (i.e., relaxation). The latter 

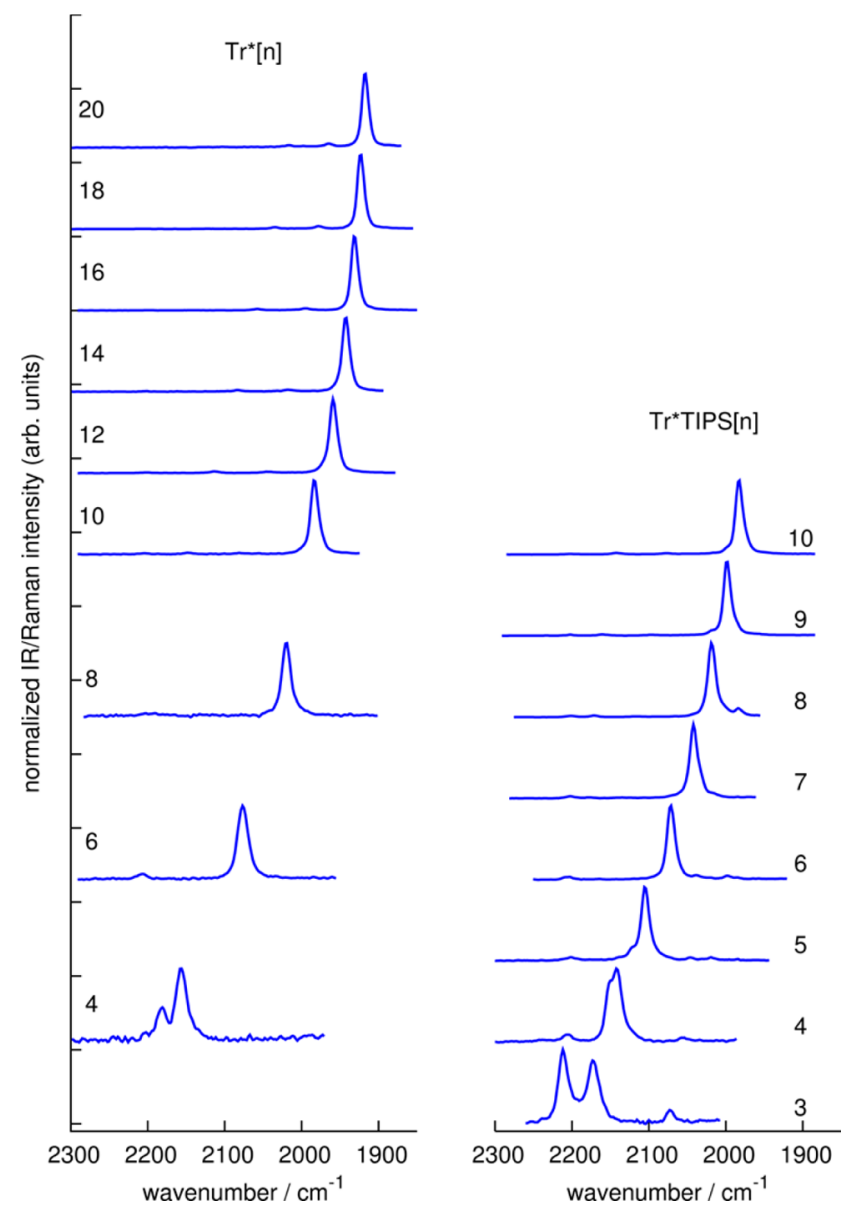

Figure 2. FT-Raman spectra of $\operatorname{Tr}^{*}[n]$ (left panel) and $\operatorname{Tr}^{*} \operatorname{TIPS}[n]$ (right panel) end-capped polyynes as measured in toluene in the range of $2300-1850 \mathrm{~cm}^{-1}$. Spectra have been normalized to the Я-peak that dominates each spectrum and provides the most significant contribution to the second hyperpolarizability (see Experimental Section for measurement conditions).

effect has been named vibrational hyperpolarizability $\left(\gamma_{\text {vib }}\right)$ and has been found ${ }^{55}$ to be directly related to intensities in Raman spectra (see below). The FT-Raman spectra of $\operatorname{Tr} *[n]$ and $\operatorname{Tr}{ }^{*} \operatorname{TIPS}[n]$, as measured in toluene, are shown in Figure 2. These spectra clearly show that the strong Raman absorption associated with the Y-line of polyynes is the dominating feature in the range of $2300-1850 \mathrm{~cm}^{-1}$. This vibration is defined as the collective in-phase stretching of triple bonds and the shrinking of single bonds (and vice versa), as shown schematically in Figure 3. ${ }^{55-60}$

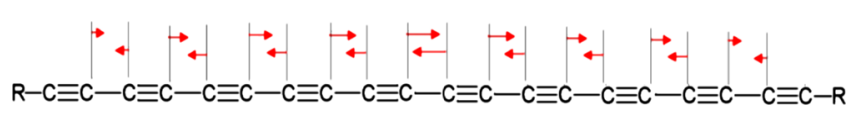

Figure 3. Schematic representation of the $Я$-mode for polyynes, resulting from the collective, in-phase stretching and shrinking of triple and single bonds (adapted from TOC graphic of ref 36).

An empirical examination of the Raman spectra shows that the Я-mode has a distinct dispersion to lower wavenumbers as a function of chain length. This feature has been recently described in detail for the $\operatorname{Tr}^{*}[\boldsymbol{n}]$ and $\operatorname{Tr}^{*} \operatorname{TIPS}[\boldsymbol{n}]$ polyynes, ${ }^{61}$ as well as other polyynes and $\pi$-conjugated systems. ${ }^{36,52,61}$ The dispersion trend versus length has been explained within the framework of the Effective Conjugation Coordinate (ECC) theory. ${ }^{62-64}$

It has been well established that for nonpolar $\pi$-conjugated systems, the vibrational contribution (i.e., nuclear relaxation contribution) to the second hyperpolarizability can be written as $^{52,55}$

$$
\left\langle\gamma_{\mathrm{vib}}\right\rangle=\frac{1}{15} \frac{1}{4 \pi^{2} c^{2}} \sum_{k} \frac{I_{k}}{\nu_{k}^{2}}
$$

where $I_{k}$ is the unpolarized absolute intensity of a Raman line found at $\nu_{k}$ wavenumbers. Thus, analysis of the vibrational contribution to the second hyperpolarizability, $\gamma_{\mathrm{vib}}$, simply reduces to the measurement of absolute Raman intensities in comparison to a standard, which is typically a selected Raman line of the solvent (see Supporting Information, Figure SI.1). Values of $\gamma_{\text {vib }}$ derived from analysis of the spectra $\operatorname{Tr}^{*}[n]$ and $\operatorname{Tr} *$ TIPS $[\boldsymbol{n}]$ polyynes using eq 2 are listed in Table 1 . The increase in $\gamma_{\text {vib }}$ as a function of polyyne length is documented for $\operatorname{Tr}^{*}[n]$ and $\operatorname{Tr} * \operatorname{TIPS}[n]$ in Figure 4, and a comparison is also added for the $\operatorname{Ad}[n]$ polyynes that have been previously measured under analogous conditions. ${ }^{52}$ The use of a semilogarithmic scale highlights the dramatic increase in nonlinear response as a function of length. While $\gamma_{\text {vib }}$ could not be obtained for $\operatorname{Tr}^{*}[2]$ because it was not synthetically available, extrapolating from the value of $\operatorname{Ad}[2]$ shows that $\gamma_{\text {vib }}{ }^{-}$ values increase by well over 3 orders of magnitude from the shortest polyynes (e.g., $\mathbf{A d}[2])$ to the longest $\mathbf{T r} *[20]$. In cases where symmetrical molecules of analogous length have been measured, a comparison of $\gamma_{\text {vib }}$-values between the $\operatorname{Ad}[n]$ and $\operatorname{Tr}^{*}[n]$ series suggests that the influence of end groups is minimal, as has also been suggested based on UV-vis spectroscopy. ${ }^{65}$ It has been established for other systems, ${ }^{66-68}$ including polyynes, ${ }^{52}$ that vibrational hyperpolarizability values $\left(\gamma_{\mathrm{vib}}\right.$, as measured here) are comparable to electronic hyperpolarizability values $\left(\gamma_{\text {ele }}\right.$, see for example TIPS $[n]$ in Table 1) in terms of both absolute value and trends when molecules within a homologous series are considered. This premise is upheld reasonably well in the case of $\operatorname{Tr}^{*}[n]$ in comparison to TIPS $[n]$ polyynes, although the values of the $\operatorname{Tr}^{*}[n]$ series are consistently somewhat higher.

It is clear from the measured data that the $\gamma_{\text {vib }}$-values of the unsymmetrical Tr*TIPS $[n]$ polyynes are higher than those of the symmetrical $\operatorname{Tr}^{*}[n]$ polyynes. This is not surprising, since polarization of a conjugated framework has been traditionally the easiest means to increase nonlinear optical response. ${ }^{69,70}$ It is interesting to note, as well, that the increased values for the $\operatorname{Tr}{ }^{*}$ TIPS $[n]$ series come with no red-shift in $\lambda_{\max }$ values, thus avoiding the transparency-nonlinearity trade-off that typically plagues organic materials. ${ }^{71,72}$

Discussion of Power Laws. It is well-known that $\gamma$-values for a series of structurally analogous oligomers will increase in a superlinear fashion as a function of length. ${ }^{69}$ The relationship between $\gamma$ and the number of repeat units, $n$, can often be modeled by a power law dependence of $\gamma \sim n^{c}$, where $c$ is the power law exponent. This trend should continue until the saturation length, or effective conjugation length (ECL), is reached, ${ }^{59,73-75}$ at which point the increase in $\gamma$ becomes monotonic versus length and exponent $c$ tends to unity. ${ }^{76}$ It has been noted by many authors that comparing the absolute $\gamma$ values for organic molecules is dangerous because the values are obtained by diverse methods, different research groups, and under various levels of resonance enhancement. ${ }^{77}$ Comparison 


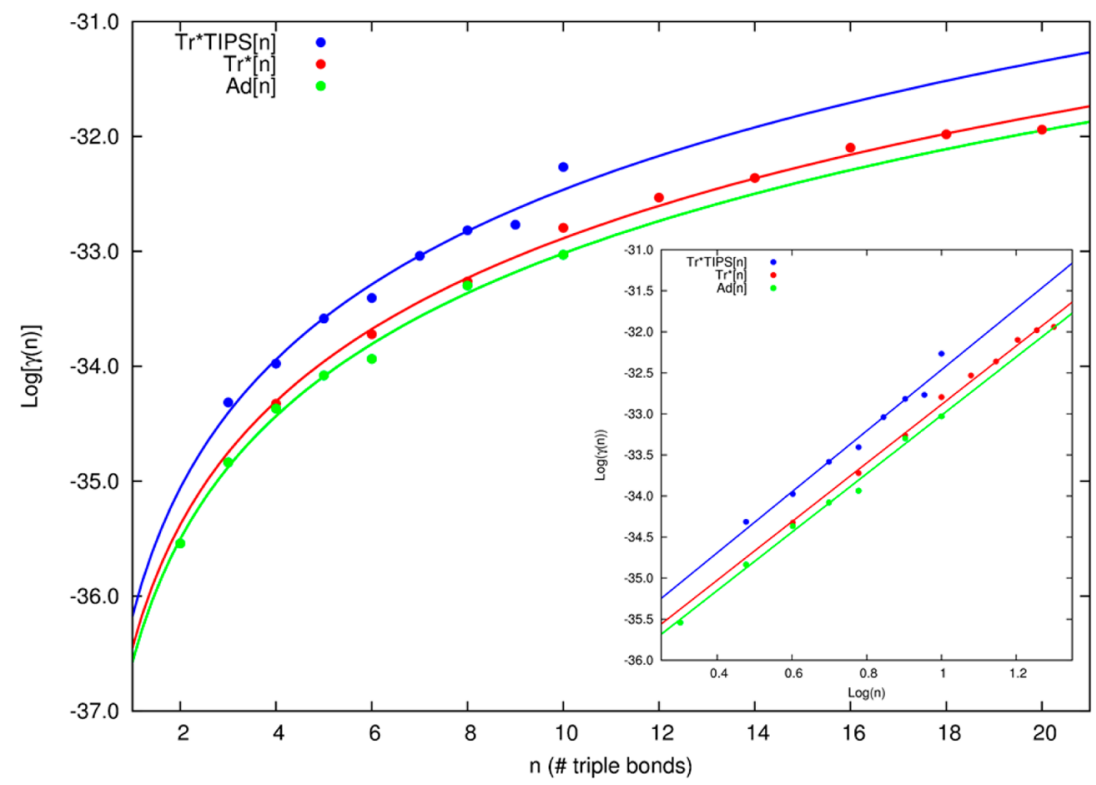

Figure 4. Vibrational contribution to the second hyperpolarizability $\left(\gamma_{\text {vib }}\right)$ versus polyyne length for $\operatorname{Tr} *[n]$ and $\operatorname{Tr} \mathbf{T r}^{*} \operatorname{TIPS}[n]$ polyynes in comparison to $\operatorname{Ad}[n]$ polyynes based on eq 1 , presented in a semilog format (inset shows same date in a log-log format).
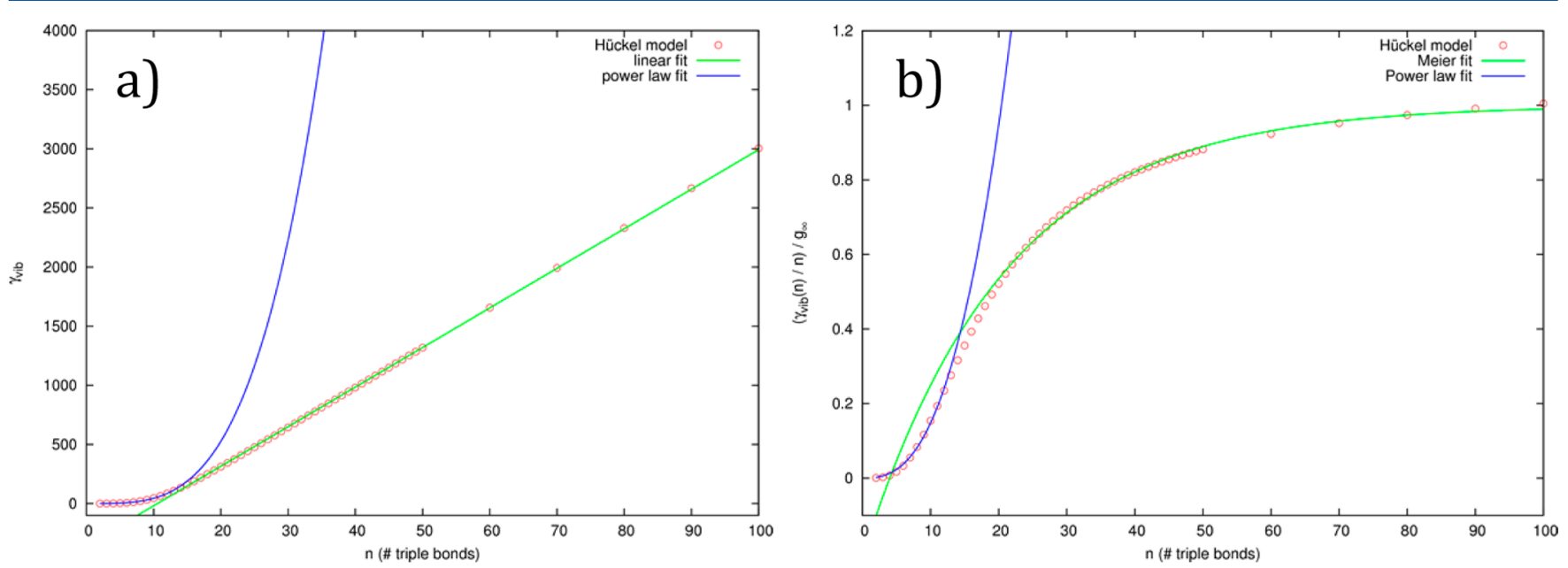

Figure 5. (a) Plot of predicted polyyne $\gamma_{\mathrm{vib}}$-values versus chain length, as computed with the Hückel theory; ${ }^{59,86}$ modeled with a power law function for small $n$-values $\left(\gamma_{\text {vib }}(n) \sim \gamma_{0} \sim n^{3.55}\right)$ and a linear fit at long chain length. (b) Plot of length independent $\gamma_{\text {vib }}(n) / n$ values versus chain length, as obtained from Hückel theory; data normalized to the specific $\gamma_{\text {vib }}$ value of carbyne, defined as $\gamma_{\infty}=\lim _{n \rightarrow \infty}\left[\gamma_{\text {vib }}(n) / n\right]$; attempted fits with both a power law and the Meier function. ${ }^{80}$

of power law exponents, on the other hand, is more straightforward, since the magnitude of power law exponent is not as susceptible to experimental differences. Thus, power law relationships are commonly used for the comparison of third-order optical nonlinearities between different series of oligomers. $^{69,73,74}$

The $\gamma_{\text {vib }}$-values for both the $\operatorname{Tr}^{*}[n]$ and $\operatorname{Tr} * \operatorname{TIPS}[n]$ polyyne series measured through the absolute Raman method show a power law relationship with approximately equal values of $c=$ 3.6, which is identical to that of the $\operatorname{Ad}[n]$ series. These values are fully in line with the power law behavior for $\gamma$ described experimentally for other polyyne series (see Figure 1 for structures), although studies are rare. The first reported study of polyynes determined that $\gamma_{\text {ele }}$ for TIPS $[n]$ polyynes shows an exponent $c=4.28,{ }^{37}$ while analysis of $\gamma_{\mathrm{ele}}$-values for phenyl endcapped polyynes $\mathbf{P h}[\boldsymbol{n}]$ gives $c=3.79 .{ }^{50}$ A series of platinum end-capped polyynes synthesized by Gladysz and co-workers, on the other hand, suggests a slightly smaller exponent $c=2.9$ (as determined by the Z-scan technique). ${ }^{48}$

In general, the power law relationships determined for polyynes $\operatorname{Tr}^{*}[n]$ and $\operatorname{Tr}{ }^{*} \operatorname{TIPS}[n]$, as well as $\operatorname{Ad}[n]$ and TIPS $[n]$, also compare quite favorably with those determined for other conjugated organic oligomers. For example, $\gamma_{\text {ele }}$-values of polytriacetylenes show a power law behavior with exponent $c$ $=2.5$ when determined by third harmonic generation (THG) and $c=2.6$ when measured by degenerate four wave mixing (DFWM). ${ }^{78,79}$ THG measurements of $\gamma$ for oligo(1,4phenyleneethynylene)s thin films give $c=3.3$ for shorter chains, ${ }^{80}$ while power law exponents of up to $c=3.6$ have been reported for polyenes (measured by electric field induced second harmonic generation, EFISH) ${ }^{73,81-83}$ Polydiacetylenes have a more intense increase versus length as measured by DFWM, in the same range as that of the polyynes with $c=4 \pm$ $0.5)^{83}$ and similarly stronger power law relationship, has been 


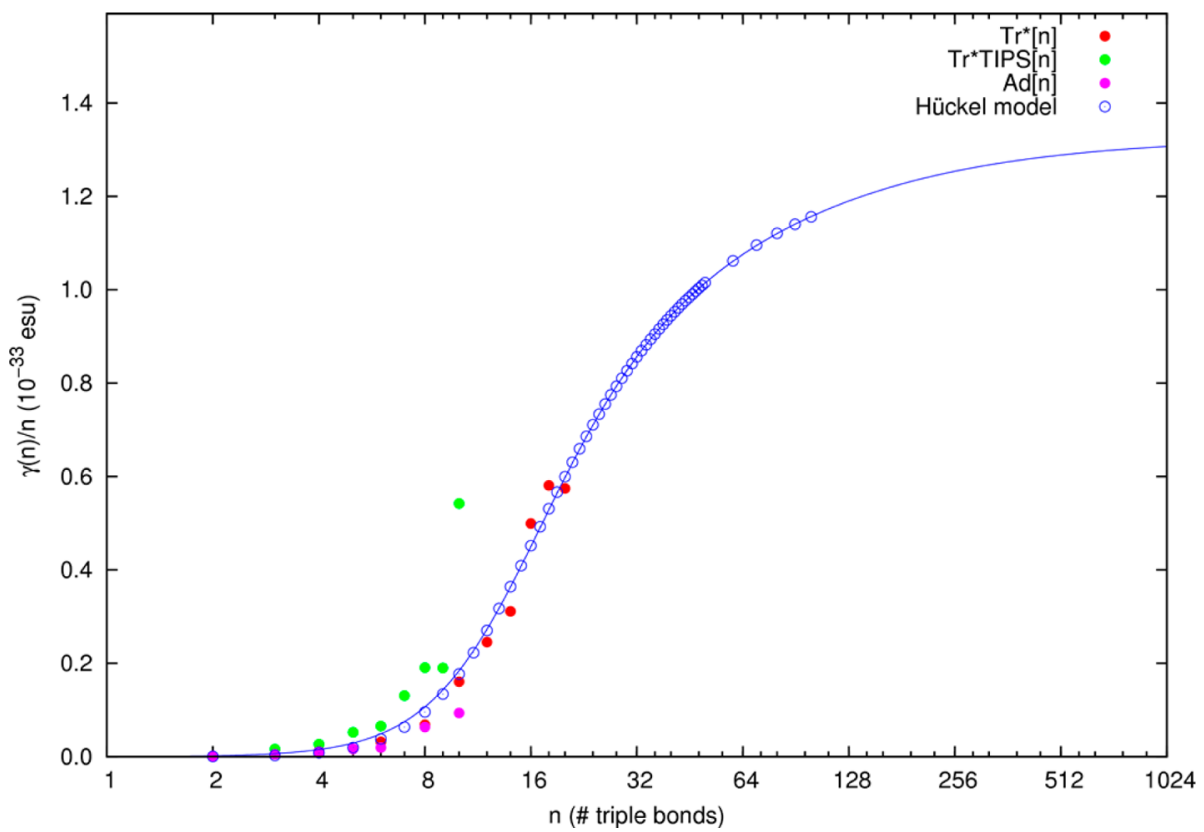

Figure 6. Plot of NLO data for $\operatorname{Ad}[n], \operatorname{Tr}^{*}[n]$, and $\operatorname{Tr}^{*}[n]$ TIPS polyynes versus length $n$ and fitting of the function $\gamma_{\infty} f(n)$ obtained from a Hückel model (see text); values of unsymmetrical, polarized $\operatorname{Tr} \mathbf{r}^{*} \operatorname{TIPS}[n]$ polyynes clearly fall away from the fit.

reported for polythiophenes evaluated by DFWM, with $c=$ $4.05 .^{84}$

Extrapolation to Carbyne. One of the most interesting aspects of the present analysis of NLO properties of $\operatorname{Tr} *[n]$ polyynes is the possibility to predict a value of the second hyperpolarizability of the allotrope carbyne. This analysis could provide an answer to the question of whether or not carbyne might be a useful NLO material. A similar analysis has been done to estimate the expected band gap of carbyne (ca. $2.6 \mathrm{eV}$ ), using experimental data for the $\operatorname{Tr}^{*}[n]$ series, ${ }^{19}$ using the estimation method reported by Meier. ${ }^{85}$ Before addressing a fit of experimental data to propose an extrapolation to carbyne, however, the $\gamma_{\text {vib }}$ of polyynes is considered using the theoretical model developed previously for the description of the Raman scattering of polyynes within a Hückel treatment, ${ }^{59,86}$ and this theoretical approximation is shown in Figure 5 (see also Supporting Information SI 3 for details). It is clear from Figure 5 that the initial increase of the predicted $\gamma_{\mathrm{vib}}$-values versus chain length follows a power law behavior for smaller values of $n$. It is interesting to note that the power law behavior has a calculated exponent of $c=3.55$, which is quite consistent with the experimental data for $\operatorname{Tr}^{*}[n], \operatorname{Tr} * \operatorname{TIPS}[n]$, and $\operatorname{Ad}[n]$ polyynes. For longer polyyne chains, the increase of $\gamma_{\mathrm{vib}}$ becomes linear (i.e., $c=1$ ). This linear behavior is consistent with achieving the ECL, as expected for the properties of a polymer, ${ }^{85}$ at the point in which the physical characteristic under consideration becomes proportional to the size of the system. With this in mind, it is thus more effective to consider the intensive counterpart (length independent) of the nonlinearity $\gamma_{\text {vib }}(n) / n$, which is achieved simply by dividing the extensive property by a quantity proportional to the size of the system (Figure 5b).

In order to extrapolate to the carbyne limit for the set of measured NLO data, it is necessary to use a fitting function that can correctly approximate the trend of $\gamma_{\text {vib }}(n)$ over the entire range of $n$ values. It is clear from inspection of Figure 5, however, that neither the power law nor the Meier function ${ }^{85}$ are able to account for the correct behavior of $\gamma_{\text {vib }}(n)$ over the complete range of experimental values. This issue has been explored using the $\gamma_{\text {vib }}(n)$ data obtained from a simple Hückel model (see Supporting Information SI 3) to develop an alternative fit function that matches the asymptotic behavior at both zero and infinity. At large $n$, the function must behave linearly, while at small $n$ it should follow a power law relationship of $\gamma_{\text {vib }} \sim n^{3.55}$, as determined experimentally for $\operatorname{Tr}^{*}[n]$. A rational function in the form of the Padé approximant eq $3,^{87}$ is a good choice to satisfy these requirements since it can be matched with the data approximation for both small and large values of $n$ :

$$
\gamma(n)=\frac{e_{4} n^{4}+e_{3} n^{3}+e_{2} n^{2}+e_{1} n+e_{0}}{f_{3} n^{3}+f_{2} n^{2}+f_{1} n+1}
$$

where $e_{k}$ and $f_{k}$ are coefficients. Indeed, at large $n$-values, eq 3 behaves linearly, as $e_{4} / f_{3} n+e_{3} / f_{3}$. On the other hand, for small $n$, the Pade approximant should agree with the known power law behavior, $n^{3.55}$, so one can impose this condition on the Padé approximant at $n=0$ by using the same derivatives of the power law $n^{3.55}$ (which are zero). Therefore, the value of Padé approximant at $n=0$, together with its first, second, and third derivatives, must vanish. This leads to the conditions:

$$
\begin{aligned}
& \gamma(0)=0 \Rightarrow e_{0}=0 \\
& \gamma^{\prime}(0)=0 \Rightarrow e_{1}=0 \\
& \gamma^{\prime \prime}(0)=0 \Rightarrow e_{2}=0 \\
& \gamma^{\prime \prime \prime}(0)=0 \Rightarrow e_{3}=0
\end{aligned}
$$

With these requirements at hand, a convenient mathematical form of a fitting law is given by a simpler Pade function of the form:

$$
\gamma(n)=\frac{e_{4} n^{4}}{f_{3} n^{3}+f_{2} n^{2}+f_{1} n+1}
$$


where $e_{4}, f_{1}, f_{2}, f_{3}$ are numerical constants to be determined. In Figure SI.2, we report the plot of $\gamma_{\text {vib }}(n)$ values determined with the Hückel model and their fitting with the Padé approximant given by eq 5 . The fitting procedure provides the following set of coefficients:

$$
\begin{aligned}
& e_{4}=6.052 \times 10^{-3} \\
& f_{1}=-1.724 \times 10^{-2} \\
& f_{2}=2.640 \times 10^{-3} \\
& f_{3}=1.757 \times 10^{-4}
\end{aligned}
$$

With the function given by eq 5 and the associated coefficients in eq 6 one can introduce a normalized fit to the quantity $\gamma_{\text {vib }}(n) / n=\mathrm{g}(n)$, such that it lies between the limiting values of 0 and 1 . The function $f(n)$ is obtained by dividing eq 5 by $n$ and then by its asymptotic limit $\left(\gamma_{\infty}=e_{4} / f_{3}\right)$. This leads to

$$
f(n)=\frac{\gamma(n) / n}{\gamma_{\infty}}=\frac{f_{3}}{e_{4}} \frac{e_{4} n^{3}}{f_{3} n^{3}+f_{2} n^{2}+f_{1} n+1}
$$

Now we can use the product $\gamma_{\infty} f(n)=\mathrm{g}(n)$ to fit the experimental NLO data to eq 7 , which have been normalized by dividing by the number of triple bonds, i.e., $\gamma_{\mathrm{vib}}(n) / n$. The only parameter that must be fit in this procedure is the asymptotic $\gamma_{\infty}$-value since the entire $f(n)$ function is now considered to be known as derived from the Hückel model. The fitting procedure just described results in the plot reported in Figure 6 , which shows that the function $f(n)$ effectively describes the behavior of the NLO data $\gamma(n) / n$ for both series of polyynes $\operatorname{Tr} *[n]$ and $\operatorname{Ad}[n]$. The function $f(n)$ in eq 7 cannot, however, be used at present to fit the NLO data for unsymmetrical polyynes Tr*TIPS $[n]$, due to the polarization effect induced by the presence of end groups with different chemical nature (and the associated changes in bond length alternation expected from symmetrically substitution). ${ }^{61}$ This is evident in the plot of $\operatorname{Tr}^{*}$ TIPS $[n] \gamma$-values in Figure 6, where the values for Tr*TIPS $[n]$ consistently fall above the fit.

The fit described by eq 7 predicts the specific optical nonlinearity for carbyne of ca. $\gamma_{\infty}=1.3 \times 10^{-33}$ esu $\left(\gamma_{\infty}=\right.$ $\left.\lim _{n \rightarrow \infty}\left[\gamma_{\mathrm{vib}}(n) / n\right]\right)$. That corresponds to a measured maximum molecular hyperpolarizability of ca. $\gamma=1.2 \times 10^{-32}$ esu $\left(\operatorname{Tr}^{*}[n], n=20\right)$. This places the expect value of carbyne within the same order of magnitude as that reported for many of the best organic materials measured under nonresonant conditions, ${ }^{88}$ such as polyenes $\left(\gamma \approx 2 \times 10^{-31}\right.$ esu, for $n=$ $25),{ }^{89}$ thiacyanines $\left(\gamma=-6.8 \times 10^{-34} \mathrm{esu}\right.$, for $\left.n=5\right),{ }^{90}$ and azathiacyanines $\left(\gamma=-9 \times 10^{-35} \mathrm{esu}\right.$, for $\left.n=4\right) .{ }^{90}$ Heflin et al. predicted that as the chains get longer, the $\gamma$-values must deviate from power law dependence and will begin to saturate. ${ }^{89}$ Our work confirms this statement with the help of calculations and long chain polyynes, which were synthesized for experimental evidence.

\section{CONCLUSIONS}

The carbon allotrope carbyne has arguably been te last of the allotropes to be explored experimentally in terms of third-order NLO properties. Thus, the most important conclusion that the present work provides is the ability to offer a prediction of the second hyperpolarizability for carbyne based on experimental data for model compound with defined length. With a prediction for $\gamma$-values of carbyne in hand, it is worth emphasizing three additional points.

(1) Even at the saturation length of carbyne (i.e., the effective conjugation length), $\gamma_{\infty}$ would be nonresonant under many experimental conditions, since the band gap for carbyne has been estimated to be rather high, at $>2.5 \mathrm{eV}$ (i.e., $\left.\lambda_{\max }<500 \mathrm{~nm}\right){ }^{19}$

(2) The current prediction for carbyne is modeled on an apolar series of molecules (i.e., $\operatorname{Tr}^{*}[\boldsymbol{n}]$ ), and it thus likely represents a lower limit of what might be achieved using polyynes as building blocks, i.e., polarized derivatives, through the addition of donor and acceptor groups, would almost certainly afford significantly enhanced $\gamma$ values, as has been established for polyenes. ${ }^{91}$ This premise is strongly supported by the present study, which shows that, while the $\operatorname{Tr}^{*}[n]$ and $\operatorname{Tr} * \operatorname{TIPS}[n]$ series show analogous power law behavior, polarization gives higher absolute $\gamma_{\text {vib }}$-values for the unsymmetrical $\operatorname{Tr} * \operatorname{TIPS}[n]$ series.

(3) The origin of the NLO response for the $\operatorname{Tr} *[n]$ polyynes derives almost exclusively from the sp-carbon framework, i.e., the Я-mode with a minimal contribution from the end groups. Thus, reducing the size of the end-capping groups should not affect $\gamma_{\infty}$ for carbyne, while the bulk nonlinearity would increase substantially as the overall molecular size decreases. This approach to increasing the bulk nonlinearity of polyynes, however, requires an alternative means to stabilize the polyyne framework, as the steric shielding influence of smaller end groups is reduced. This might be accomplished through the use of mechanical bonds, which provide polyyne rotaxanes in which the sp-carbon framework is shielded by a macrocycle, without covalent modifications to the polyyne framework. Such systems are now under study. ${ }^{92,93}$

\section{ASSOCIATED CONTENT}

\section{Supporting Information}

The Supporting Information is available free of charge on the ACS Publications website at DOI: 10.1021/acs.jpcc.6b03071.

Additional details and figures as described in the text (PDF)

\section{AUTHOR INFORMATION}

\section{Corresponding Author}

*E-mail: nisha.rani.agarwal@gmail.com.

\section{Present Address}

"Chalmers University of Technology, Gothenburg, Sweden. Telephone number: + 46 (0)31 7723442 .

Notes

The authors declare no competing financial interest.

\section{ACKNOWLEDGMENTS}

M.T. and N.R.A thank the Italian "Ministero dell'Istruzione, dell'Università e della Ricerca” (MIUR) for financial support, under the auspices of the FIRB program RBFR08XH0H (Futuro in Ricerca 2008). R.R.T. thanks the Deutsche Forschungsgemeinschaft (DFG SFB 953, Synthetic Carbon Allotropes) and NSERC for financial support of this work. We acknowledge Elena Rodighiero for her involvement, during her 
Master Thesis, ${ }^{94}$ with the development of the Hückel model described here.

\section{REFERENCES}

(1) Kratschmer, W.; Lamb, L. D.; Fostiropoulos, K.; Huffman, D. R. Solid $\mathrm{C}_{60}$ : a new form of carbon. Nature 1990, 347, 354-358.

(2) Hirsch, A. The era of carbon allotropes. Nat. Mater. 2010, 9, $868-871$.

(3) Falcao, E. H. L.; Wudl, F. Carbon allotropes: beyond graphite and diamond. J. Chem. Technol. Biotechnol. 2007, 82, 524-531.

(4) Diederich, F. Carbon scaffolding: building acetylenic all-carbon and carbon-rich compounds. Nature (London, U. K.) 1994, 369, 199207.

(5) Diederich, F.; Rubin, Y. Synthetic approaches toward molecular and polymeric carbon allotropes. Angew. Chem., Int. Ed. Engl. 1992, 31, $1101-1123$.

(6) Casari, C. S.; Tommasini, M.; Tykwinski, R. R.; Milani, A. Carbon-atom wires: 1-D systems with tunable properties. Nanoscale 2016, 8, 4414-4435.

(7) Schwertfeger, H.; Fokin, A. A.; Schreiner, P. R. Diamonds are a chemist's best friend: diamondoid chemistry beyond adamantane. Angew. Chem., Int. Ed. 2008, 47, 1022-1036.

(8) Hsu, C. H.; Xu, J. Diamond nanowire - a challenge from extremes. Nanoscale 2012, 4, 5293-5299.

(9) Liu, T.; Troisi, A. What makes fullerene acceptors special as electron acceptors in organic solar cells and how to replace them. Adv. Mater. 2013, 25, 1038-1041.

(10) Park, H.; Afzali, A.; Han, S. J.; Tulevski, G. S.; Franklin, A. D.; Tersoff, J.; Hannon, J. B.; Haensch, W. High-density integration of carbon nanotubes via chemical self-assembly. Nat. Nanotechnol. 2012, 7, 787-791.

(11) Bae, S.; Kim, H.; Lee, Y.; Xu, X. F.; Park, J. S.; Zheng, Y.; Balakrishnan, J.; Lei, T.; Kim, H. R.; Song, Y. I.; et al. Roll-to-roll production of 30-in. graphene films for transparent electrodes. Nat. Nanotechnol. 2010, 5, 574-578.

(12) Malko, D.; Neiss, C.; Vines, F.; Gorling, A. Competition for graphene: graphynes with direction-dependent Dirac cones. Phys. Rev. Lett. 2012, 108, 086804.

(13) Tykwinski, R. R. Carbyne: The molecular approach. Chem. Rec. 2015, 15, 1060-1074.

(14) Lagow, R. J.; Kampa, J. J.; Wei, H.-C.; Battle, S. L.; Genge, J. W.; Laude, D. A.; Harper, C. J.; Bau, R.; Stevens, R. C.; Haw, J. F.; et al. Synthesis of linear acetylenic carbon: The "sp" carbon allotrope. Science (Washington, DC, U. S.) 1995, 267, 362-367.

(15) Cataldo, F. A study on the structure and electrical properties of the fourth carbon allotrope: carbine. Polym. Int. 1997, 44, 191-200.

(16) Polyynes: Synthesis, Properties, and Applications; Cataldo, F., Ed.; Taylor \& Francis: Boca Raton, FL, 2006.

(17) Carbyne and Carbynoid Structures; Heimann, R. B., Evsyukov, S. E., Kavan, L., Eds.; Kluwar Academic Press: London, 1999.

(18) Chalifoux, W. A.; Tykwinski, R. R. Synthesis of extended polyynes: Toward carbyne. C. R. Chim. 2009, 12, 341-358.

(19) Chalifoux, W. A.; Tykwinski, R. R. Synthesis of polyynes to model the sp-carbon allotrope carbine. Nat. Chem. 2010, 2, 967-971.

(20) Tsuji, M.; Kuboyama, S.; Matsuzaki, T.; Tsuji, T. Formation of hydrogen-capped polyynes by laser ablation of $\mathrm{C}_{60}$ particles suspended in solution. Carbon 2003, 41, 2141-2148.

(21) Lucotti, A.; Tommasini, M.; Del Zoppo, M.; Castiglioni, C.; Zerbi, G.; Cataldo, F.; Casari, C. S.; Li Bassi, A.; Russo, V.; Bogana, M.; et al. Raman and SERS investigation of isolated sp carbon chains. Chem. Phys. Lett. 2006, 417, 78-82.

(22) Tabata, H.; Fujii, M.; Hayashi, S. Laser ablation of diamond nanoparticles suspended in solvent: synthesis of polyynes. Chem. Phys. Lett. 2004, 395, 138-142.

(23) Casari, C. S.; Russo, V.; Li Bassi, A.; Bottani, C. E.; Cataldo, F.; Lucotti, A.; Tommasini, M.; Del Zoppo, M.; Castiglioni, C.; Zerbi, G. Stabilization of linear carbon structures in a solid $\mathrm{Ag}$ nanoparticle assembly. Appl. Phys. Lett. 2007, 90, 013111.
(24) Zhao, X.; Ando, Y.; Liu, Y.; Jinno, M.; Suzuki, T. Carbon nanowire made of a long linear carbon chain inserted inside a multiwalled carbon nanotube. Phys. Rev. Lett. 2003, 90, 187401.

(25) Nishide, D.; Dohi, H.; Wakabayashi, T.; Nishibori, E.; Aoyagi, S.; Ishida, M.; Kikuchi, S.; Kitaura, R.; Sugai, T.; Sakata, M.; et al. Single-wall carbon nanotubes encaging linear chain $\mathrm{C}_{10} \mathrm{H}_{2}$ polyyne molecules inside. Chem. Phys. Lett. 2006, 428, 356-360.

(26) Wakabayashi, T.; Ong, A.-L.; Strelnikov, D.; Krätschmer, W. Flashing carbon on cold surfaces. J. Phys. Chem. B 2004, 108, 36863690.

(27) Strelnikov, D.; Reusch, R.; Krätschmer, W. Assignment of carbon chain molecules in cryogenic matrices by selective laserinduced oxidation. J. Phys. Chem. A 2005, 109, 7708-7713.

(28) Casari, C. S.; Li Bassi, A.; Ravagnan, L.; Siviero, F.; Lenardi, C.; Piseri, P.; Bongiorno, G.; Bottani, C. E.; Milani, P. Chemical and thermal stability of carbyne-like structures in cluster-assembled carbon films. Phys. Rev. B: Condens. Matter Mater. Phys. 2004, 69, 075422.

(29) Heymann, D. Thermolysis of the polyyne $\mathrm{C}_{8} \mathrm{H}_{2}$ in hexane and methanol: Experimental and theoretical study. Carbon 2005, 43, 2235-2242.

(30) Baughman, R. H. Chemistry. Dangerously seeking linear carbon. Science 2006, 312, 1009-1110.

(31) Jahnke, E.; Tykwinski, R. R. The Fritsch-Buttenberg-Wiechell rearrangement: modern applications for an old reaction. Chem. Commun. 2010, 46, 3235-3249.

(32) Chalifoux, W. A.; Tykwinski, R. R. Polyyne synthesis using carbene/carbenoid rearrangements. Chem. Rec. 2006, 6, 169-182.

(33) Chalifoux, W. A.; Ferguson, M. J.; Tykwinski, R. R. Tris(biphenyl-4-yl)silyl-endcapped polyynes. Eur. J. Org. Chem. 2007, 2007, 1001-1006.

(34) Chalifoux, W. A.; McDonald, R.; Ferguson, M. J.; Tykwinski, R. $\mathrm{R}$ tert-Butyl-End-Capped Polyynes: Crystallographic evidence of reduced bond-length alternation. Angew. Chem., Int. Ed. 2009, 48, 7915-7919.

(35) Chalifoux, W. A.; Ferguson, M. J.; McDonald, R.; Melin, F.; Echegoyen, L.; Tykwinski, R. R. Adamantyl-endcapped polyynes. J. Phys. Org. Chem. 2012, 25, 69-76.

(36) Lucotti, A.; Tommasini, M.; Fazzi, D.; Del Zoppo, M.; Chalifoux, W. A.; Ferguson, M. J.; Zerbi, G.; Tykwinski, R. R. Evidence for solution-state nonlinearity of sp-carbon chains based on IR and Raman spectroscopy: Violation of mutual exclusion. J. Am. Chem. Soc. 2009, 131, 4239-4244.

(37) Eisler, S.; Slepkov, A. D.; Elliott, E.; Luu, T.; McDonald, R.; Hegmann, F. A.; Tykwinski, R. R. Polyynes as a model for carbyne: Synthesis, physical properties, and nonlinear optical response. J. Am. Chem. Soc. 2005, 127, 2666-2676.

(38) Szafert, S.; Gladysz, J. A. Carbon in one dimension: Structural analysis of the higher conjugated polyynes. Chem. Rev. 2003, 103, 4175-4206; Chem. Rev. 2006, 106, PR1-PR33.

(39) Tobe, Y.; Wakabayashi, T. In Acetylene Chemistry: Chemistry, Biology, and Material Science; Diederich, F., Stang, P. J., Tykwinski, R. R, Eds.; Wiley-VCH: Weinheim, Germany, 2005; Chapter 9.

(40) Wang, C.; Batsanov, A. S.; West, K.; Bryce, M. R. Synthesis and crystal structures of isolable terminal aryl hexatriyne and octatetrayne derivatives: $\mathrm{Ar}-(\mathrm{C} \equiv \mathrm{C})_{\mathrm{n}} \mathrm{H}(\mathrm{n}=3$, 4). Org. Lett. 2008, 10, 3069-3072.

(41) Simpkins, S. M. E.; Weller, M. D.; Cox, L. R. $\beta$ Chlorovinylsilanes as masked alkynes in oligoyne assembly: synthesis of the first aryl-end-capped dodecayne. Chem. Commun. 2007, 40354037.

(42) Zheng, Q.; Bohling, J. C.; Peters, T. B.; Frisch, A. C.; Hampel, F.; Gladysz, J. A. A Synthetic breakthrough into an unanticipated stability regime: $A$ series of isolable complexes in which $\mathrm{C}_{6}, \mathrm{C}_{8}, \mathrm{C}_{10}$, $\mathrm{C}_{12}, \mathrm{C}_{16}, \mathrm{C}_{20}, \mathrm{C}_{24}$, and $\mathrm{C}_{28}$ polyynediyl chains span two Platinum atoms. Chem. - Eur. J. 2006, 12, 6486-6505.

(43) Antonova, A. B.; Bruce, M. I.; Elis, B. G.; Gaudio, M.; Humphrey, P. A.; Jevric, M.; Melino, G.; Nicholson, B. K.; Perkins, G. J.; Skelton, B. W.; et al. A novel methodology for the synthesis of complexes containing long carbon chains linking metal centres: 
molecular structures of $\left\{\mathrm{Ru}(\mathrm{dppe}) \mathrm{Cp}^{*}\right\}_{2}\left(\mu-\mathrm{C}_{14}\right)$ and $\left\{\mathrm{Co}_{3}(\mu\right.$-dppm $)$ (CO) $)_{7}\left(\mu_{3}: \mu_{3}-\mathrm{C}_{16}\right)$. Chem. Commun. 2004, 960-961.

(44) Eastmond, R.; Johnson, T. R.; Walton, D. R. M. Silylation as a protective method for terminal alkynes in oxidative couplings: A general synthesis of the parent polyynes $\mathrm{H}(\mathrm{C} \equiv \mathrm{C})_{\mathrm{n}} \mathrm{H}(\mathrm{n}=4-10,12)$. Tetrahedron 1972, 28, 4601-4616.

(45) Arendt, A.; Kolkowski, R.; Samoc, M.; Szafert, S. Spectral dependence of nonlinear optical properties of symmetrical octatetraynes with p-substituted phenyl end-groups. Phys. Chem. Chem. Phys. 2015, 17, 13680-13688.

(46) Fazio, E.; D’Urso, L.; Consiglio, G.; Giuffrida, A.; Compagnini, G.; Puglisi, O.; Patanè, S.; Neri, F.; Forte, G. Nonlinear scattering and absorption effects in size-selected diphenylpolyynes. J. Phys. Chem. C 2014, 118, 28812-28819.

(47) Frank, B. B.; Laporta, P. R.; Breiten, B.; Kuzyk, M. C.; Jarowski, P. D.; Schweizer, W. B.; Seiler, P.; Biaggio, I.; Boudon, C.; Gisselbrecht, J.-P.; et al. Comparison of CC triple and double bonds as spacers in push-pull chromophores. Eur. J. Org. Chem. 2011, 2011, 4307-4317.

(48) Samoc, M.; Dalton, G. T.; Gladysz, J. A.; Zheng, Q.; Velkov, Y.; Agren, H.; Norman, P.; Humphrey, M. G. Cubic nonlinear optical properties of Platinum-terminated polyynediyl chains. Inorg. Chem. 2008, 47, 9946-9957.

(49) Slepkov, A. D.; Hegmann, F. A.; Eisler, S.; Elliott, E.; Tykwinski, R. R. The surprising nonlinear optical properties of conjugated polyyne oligomers. J. Chem. Phys. 2004, 120, 6807-6810.

(50) Luu, T.; Elliott, E.; Slepkov, A. D.; Eisler, S.; McDonald, R.; Hegmann, F. A.; Tykwinski, R. R. Synthesis, structure, and nonlinear optical properties of diarylpolyynes. Org. Lett. 2005, 7, 51-54.

(51) Perry, J. W. Nonlinear optical properties of molecules and materials. ACS Symp. Ser. 1991, 455, 67-88.

(52) Lucotti, A.; Tommasini, M.; Fazzi, D.; Del Zoppo, M.; Chalifoux, W. A.; Tykwinski, R. R.; Zerbi, G. Absolute Raman intensity measurements and determination of the vibrational second hyperpolarizability of adamantyl endcapped polyynes. J. Raman Spectrosc. 2012, 43, 1293-1298.

(53) Slepkov, A. D.; Hegmann, F. A.; Zhao, Y.; Tykwinski, R. R.; Kamada, K. Ultrafast optical Kerr effect measurements of third-order nonlinearities in cross-conjugated iso-polydiacetylene oligomers. $J$. Chem. Phys. 2002, 116, 3834-3840.

(54) Shafei, S.; Kuzyk, M. G. Critical role of the energy spectrum in determining the nonlinear-optical response of a quantum system. J. Opt. Soc. Am. B 2011, 28, 882-891.

(55) Castiglioni, C.; Gussoni, M.; Del Zoppo, M.; Zerbi, G. Relaxation contribution to hyperpolarizability. A semiclassical model. Solid State Commun. 1992, 82, 13-17.

(56) Milani, A.; Tommasini, M.; Zerbi, G. Connection among Raman wavenumbers, bond length alternation and energy gap in polyynes. J. Raman Spectrosc. 2009, 40, 1931-1934.

(57) Milani, A.; Tommasini, M.; Fazzi, D.; Castiglioni, C.; Del Zoppo, M.; Zerbi, G. First-principles calculation of the Peierls distortion in an infinite linear carbon chain: the contribution of Raman spectroscopy. J. Raman Spectrosc. 2008, 39, 164-168.

(58) Milani, A.; Tommasini, M.; Zerbi, G. Carbynes phonons: A tight binding force field. J. Chem. Phys. 2008, 128, 064501.

(59) Tommasini, M.; Milani, A.; Fazzi, D.; Del Zoppo, M.; Castiglioni, C.; Zerbi, G. Modeling phonons of carbon nanowires. Phys. E 2008, 40, 2570-2576.

(60) Tommasini, M.; Fazzi, D.; Milani, A.; Del Zoppo, M.; Castiglioni, C.; Zerbi, G. Intramolecular vibrational force fields for linear carbon chains through an adaptative linear scaling scheme. $J$. Phys. Chem. A 2007, 111, 11645-11651.

(61) Agarwal, N. R.; Lucotti, A.; Fazzi, D.; Tommasini, M.; Castiglioni, C.; Chalifoux, W. A.; Tykwinski, R. R. Structure and chain polarization of long polyynes investigated with infrared and Raman spectroscopy. J. Raman Spectrosc. 2013, 44, 1398-1410.

(62) Castiglioni, C.; Tommasini, M.; Zerbi, G. Raman spectroscopy of polyconjugated molecules and materials: confinement effect in one and two dimensions. Philos. Trans. R. Soc., A 2004, 362, 2425-2459.
(63) Del Zoppo, M.; Castiglioni, C.; Zuliani, P.; Zerbi, G. In Handbook of Conducting Polymers, 2nd ed.; Skotheim, T., Ed.; Dekker: New York, 1998.

(64) Castiglioni, C.; Del Zoppo, M.; Zerbi, G. Vibrational Raman spectroscopy of polyconjugated organic oligomers and polymers. J. Raman Spectrosc. 1993, 24, 485-494.

(65) Tykwinski, R. R.; Chalifoux, W. A.; Eisler, S. R.; Lucotti, A.; Tommasini, M.; Fazzi, D.; Del Zoppo, M.; Zerbi, G. Toward carbyne: Synthesis and stability of really long polyynes. Pure Appl. Chem. 2010, $82,891-904$.

(66) Rumi, M.; Zerbi, G.; Müllen, K.; Müller, G.; Rehahn, M. Nonlinear-optical and vibrational properties of conjugated polyaromatic molecules. J. Chem. Phys. 1997, 106, 24-34.

(67) Castiglioni, C.; Del Zoppo, M.; Zerbi, G. Molecular first hyperpolarizability of push-pull polyenes: Relationship between electronic and vibrational contribution by a two-state model. Phys. Rev. B: Condens. Matter Mater. Phys. 1996, 53, 13319.

(68) Del Zoppo, M.; Castiglioni, C.; Zuliani, P.; Zerbi, G. NLO responses of organic materials: The vibrational approach. Adv. Mater. 1996, 8, 345-347.

(69) Meier, H. Conjugated oligomers with terminal donor-acceptor substitution. Angew. Chem., Int. Ed. 2005, 44, 2482-2506.

(70) Tykwinski, R. R.; Gubler, U.; Martin, R. E.; Diederich, F.; Bosshard, C.; Gunter, P. Structure-property relationships in thirdorder nonlinear optical chromophores. J. Phys. Chem. B 1998, 102, 4451-4465.

(71) Ledoux, I.; Zyss, J.; Jutand, A.; Amatore, C. Nonlinear optical properties of asymmetric polyphenyls: Efficiency versus transparency trade-off. Chem. Phys. 1991, 150, 117-123.

(72) Moylan, C. R.; Twieg, R. J.; Lee, V. Y.; Swanson, S. A.; Betterton, K. M.; Miller, R. D. Nonlinear optical chromophores with large hyperpolarizabilities and enhanced thermal stabilities. J. Am. Chem. Soc. 1993, 115, 12599-12600.

(73) Bubeck, C. In Electronic Materials: The Oligomer Approach; Müllen, K., Wegner, G., Eds.; Wiley-VCH: Weinheim, Germany, 1998; Chapter 8.

(74) Brédas, J.-L.; Adant, C.; Tackx, P.; Persoons, A.; Pierce, B. M. Third-order nonlinear optical response in organic materials: Theoretical and experimental aspects. Chem. Rev. 1994, 94, 243-278.

(75) Gubler, U.; Bosshard, C. Molecular design for third-order nonlinear optics. Adv. Polym. Sci. 2002, 158, 123-191.

(76) Samuel, I. D. W.; Ledoux, I.; Dhenaut, C.; Zyss, J.; Fox, H. H.; Schrock, R. R.; Silbey, R. J. Saturation of cubic optical nonlinearity in long-chain polyene oligomers. Science (Washington, DC, U. S.) 1994, 265, 1070-1072.

(77) Willetts, A.; Rice, J. E.; Burland, D. M.; Shelton, D. P. Problems in the comparison of theoretical and experimental hyperpolarizabilities. J. Chem. Phys. 1992, 97, 7590-7599.

(78) Martin, R. E.; Gubler, U.; Boudon, C.; Gramlich, V.; Bosshard, C.; Gisselbrecht, J.-P.; Günter, P.; Gross, M.; Diederich, F. Characterization, and estimation of the effective conjugation length by llectrochemical, UV/Vis, and nonlinear optical methods. Chem. Eur. J. 1997, 3, 1505-1512.

(79) Gubler, U.; Bosshard, C.; Günter, P.; Balakina, M. Y.; Cornil, J.; Bredas, J. L.; Martin, R. E.; Diederich, F. Scaling law for second-order hyperpolarizability in poly(triacetylene) molecular wires. Opt. Lett. 1999, 24, 1599-1601.

(80) Meier, H.; Ickenroth, D.; Stalmach, U.; Koynov, K.; Bahtiar, A.; Bubeck, C. Preparation and nonlinear optics of monodisperse oligo(1,4-phenyleneethynylene)s. Eur. J. Org. Chem. 2001, 2001, 4431-4443.

(81) Nalwa, H. S. In Nonlinear Optics of Organic Molecules and Polymers; Nalwa, H. S., Miyata, S.; Eds.; CRC Press: Boca Raton, FL, 1997; Chapters 9 and 11.

(82) Craig, G. S. W.; Cohen, R. E.; Schrock, R. R.; Silbey, R. J.; Puccetti, G.; Ledoux, I.; Zyss, J. Nonlinear optical analysis of a series of triblock copolymers containing model polyenes: the dependence of hyperpolarizability on conjugation length. J. Am. Chem. Soc. 1993, 115, $860-867$. 
(83) Byrne, H. J.; Blau, W.; Giesa, R.; Schulz, R. C. Nonlinear optical studies of graded enyne oligomers. Chem. Phys. Lett. 1990, 167, 484489.

(84) Zhao, M.-T.; Singh, B. P.; Prasad, P. N. A systematic study of polarizability and microscopic third-order optical nonlinearity in thiophene oligomers. J. Chem. Phys. 1988, 89, 5535-5541.

(85) Meier, H.; Stalmach, U.; Kolshorn, H. Effective conjugation length and UV/vis spectra of oligomers. Acta Polym. 1997, 48, 379384.

(86) Tommasini, M.; Fazzi, D.; Milani, A.; Del Zoppo, M.; Castiglioni, C.; Zerbi, G. Effective hamiltonian for $\pi$ electrons in linear carbon chains. Chem. Phys. Lett. 2007, 450, 86-90.

(87) Baker, G. A.; Graves-Morris, P. R. Padé Approximants: XD-US.; Cambridge University Press: Cambridge, U.K., 1996.

(88) Kuzyk, M. G. Using fundamental principles to understand and optimize nonlinear-optical materials. J. Mater. Chem. 2009, 19, 74447465.

(89) Heflin, J. R.; Wong, K. Y.; Zamani-Khamiri, O.; Garito, A. F. Nonlinear optical properties of linear chains and electron-correlation effects. Phys. Rev. B: Condens. Matter Mater. Phys. 1988, 38, 15731576.

(90) Werncke, W.; Pfeiffer, M.; Lau, A.; Grahn, W.; Johannes, H.-H.; Dähne, L. Alternation and saturation effects in the chain-length dependences of the third-order hyperpolarizabilities of cyanines. J. Opt. Soc. Am. B 1998, 15, 863-870.

(91) Marder, S. R.; Torruellas, W. E.; Blanchard-Desce, M.; Ricci, V.; Stegeman, G. I.; Gilmour, S.; Brédas, J.-L.; Li, J.; Bublitz, G. U.; Boxer, S. G. Large molecular third-order optical nonlinearities in polarized carotenoids. Science (Washington, DC, U. S.) 1997, 276, 1233-1236.

(92) Movsisyan, L. D.; Kondratuk, D. V.; Franz, M.; Thompson, A. L.; Tykwinski, R. R.; Anderson, H. L. Synthetic analogues of the active site of the A-cluster of acetyl coenzyme A synthase/CO dehydrogenase: Syntheses, structures, and reactions with CO. Org. Lett. 2012, 14, 3424-3436.

(93) Movsisyan, L.; Franz, M.; Hampel, F.; Thompson, A.; Tykwinski, R. R.; Anderson, H. L. Polyyne rotaxanes: Stabilization by encapsulation. J. Am. Chem. Soc. 2016, 138, 1366-1376.

(94) Rodighiero, E. Master Thesis in Nuclear Engineering, Politecnico di Milano, Milano, Italy, 2008. 